\title{
A Reconfigurable Antenna with Beam Steering and Beamwidth Variability for Wireless Communications
}

\author{
Md. Asaduzzaman Towfiq, Student, IEEE, Israfil Bahceci, Member, IEEE, Sebastián Blanch, \\ Jordi Romeu, Fellow, IEEE, Lluís Jofre, Fellow, IEEE, and Bedri A. Cetiner, Member, IEEE
}

\begin{abstract}
A reconfigurable antenna (RA) capable of steering its beam into the hemisphere corresponding to $\theta \in$ $\left\{-40^{\circ}, 0^{\circ}, 40^{\circ}\right\}, \phi \in\left\{0^{\circ}, 45^{\circ}, 90^{\circ},-45^{\circ}\right\}$, and of changing 3dB beamwidth, where $\theta_{3 d B} \in\left(40^{\circ}, 100^{\circ}\right), \phi \in\left\{45^{\circ}, 90^{\circ},-45^{\circ}\right\}$ for broadside direction is presented. The RA operating in 5 GHz band consists of a driven patch antenna with a parasitic layer placed above it. The upper surface of the parasitic layer has two pixelated metallic strips, where each strip has four pixels. The pixels connected via PIN diode switches enable to change the current distribution on the antenna providing the desired modes of operation. A prototype RA was characterized indicating an average gain of $8 \mathrm{~dB}$. Measured and simulated impedance and radiation patterns agreed well. The proposed RA offers an efficient solution by using less number of switches compared to other RAs. The system level simulations for a 5G orthogonal frequency division multiple access system show that the RA improves capacity/coverage trade-off significantly, where the RA modes and users are jointly determined to create proper beamwidth and directivity at the access point antennas. For a hotspot scenario, the presented RA provided $29 \%$ coverage and $16 \%$ capacity gain concurrently.
\end{abstract}

Index Terms-Reconfigurable antennas, beam steering, parasitic antennas, antenna radiation patterns, antenna diversity

\section{INTRODUCTION}

$\mathbf{I}$ NCREASING demand for high data rate and extreme broadband wireless services along with scarcity of frequency spectrum and explosive user growth have prompted researchers to explore suitable methodologies to optimize the use of the wireless medium. Reconfigurable antennas (RAs) capable of dynamically changing their properties, e.g., radiation pattern, operational frequency and polarization have received a great deal of attention [1]-[12]. In particular, the RAs with radiation pattern reconfigurability help improve the system performance [13], communication security and energy efficiency by directing signals towards intended directions while reducing interference in unintended directions [14].

This work is supported in part by AFOSR Grant No FA 9550-15-10040DEF, and Defense University Research Instrumentation Program (DURIP) Grant No: FA9550-16-1-0352

Md. A. Towfiq and B. Cetiner are with the Department of Electrical and Computer Engineering, Utah State University, Logan, UT, 84322, and i5 Technologies, Inc., North Logan UT, 84341. e-mails: \{aztowfiq@aggiemail.usu.edu,bedri.cetiner@usu.edu\}.

I. Bahceci is with Ericsson Canada, Kanata, ON, Canada, K2K2R9. e-mail: israfil.bahceci@ericsson.com.

S. Blanch, L. Jofre, and J. Romeu are with the AntennaLab Research Group, Department of Signal Theory and Communications, Universitat Politècnica de Catalunya (UPC), 08034 Barcelona, Spain (e-mails: \{jofre,romeu\}@tsc.upc.edu)
The gains with RA systems are typically achieved at the cost of increased structural (or hardware) and algorithmic (or software) complexities as compared to a legacy singlefunction antenna system. Therefore, it is critical to design and implement an RA equipped communication system where the hardware and software complexities are kept at minimal, and this is the main motivation behind this work. An RA system that is capable of joint beam-steering and beamwidth control enables the network to respond to spatial user variations in a dynamical manner, which is prominent for many $5 \mathrm{G}$ use case scenarios [15]. In particular, hot-spot and ultra dense deployment, and the associated spatia-temporal user and traffic variations for typical 5G technologies [16]-[18] require the radio access network to dynamically control its coverage area [19]-[21]. The RA of this work facilitates the creation of a multitude of element radiation patterns that can be used to reshape the coverage area as desired in accordance with the network and channel variations.

There are a number of approaches for accomplishing antenna reconfigurability [22]-[32]. The approach adopted in [30]-[32] provides an efficient solution by separating the driven antenna from a reconfigurable parasitic layer. In [30], a reconfigurable parasitic pixel layer placed in the near-field of the driven antenna is utilized to change the reactive loading providing nine different beam steering modes. This work introduced a relatively simple method for pattern reconfiguration, albeit with a large number of switches (e.g., at least 12 PIN diodes) resulting in manufacturing complexity and reduced antenna gain due to the ohmic losses of diodes. Another approach to control antenna pattern is to employ partially reflective surface (PRS) in the vicinity of a driven antenna. It is shown in [33]-[35] that the beamwidth of the antennas employing PRSs can be dynamically controlled by changing the reflective properties of the surface. These antennas offer high gain at the cost of using a large number of varactor diodes to tune the surface impedance. While several designs exist for beam steering, a few work has been carried out to perform beam steering and beamwidth variability simultaneously on a single antenna architecture [9], [36], [37].

The RA presented in this work employs a structure similar to the one in [30] and is capable of both beam-steering and beamwidth control while requiring a small number of switches, where only six switches are used. This RA can steer its main beam into nine different directions and achieve three distinct $3-\mathrm{dB}$ beamwidths in three planes corresponding to a total of 

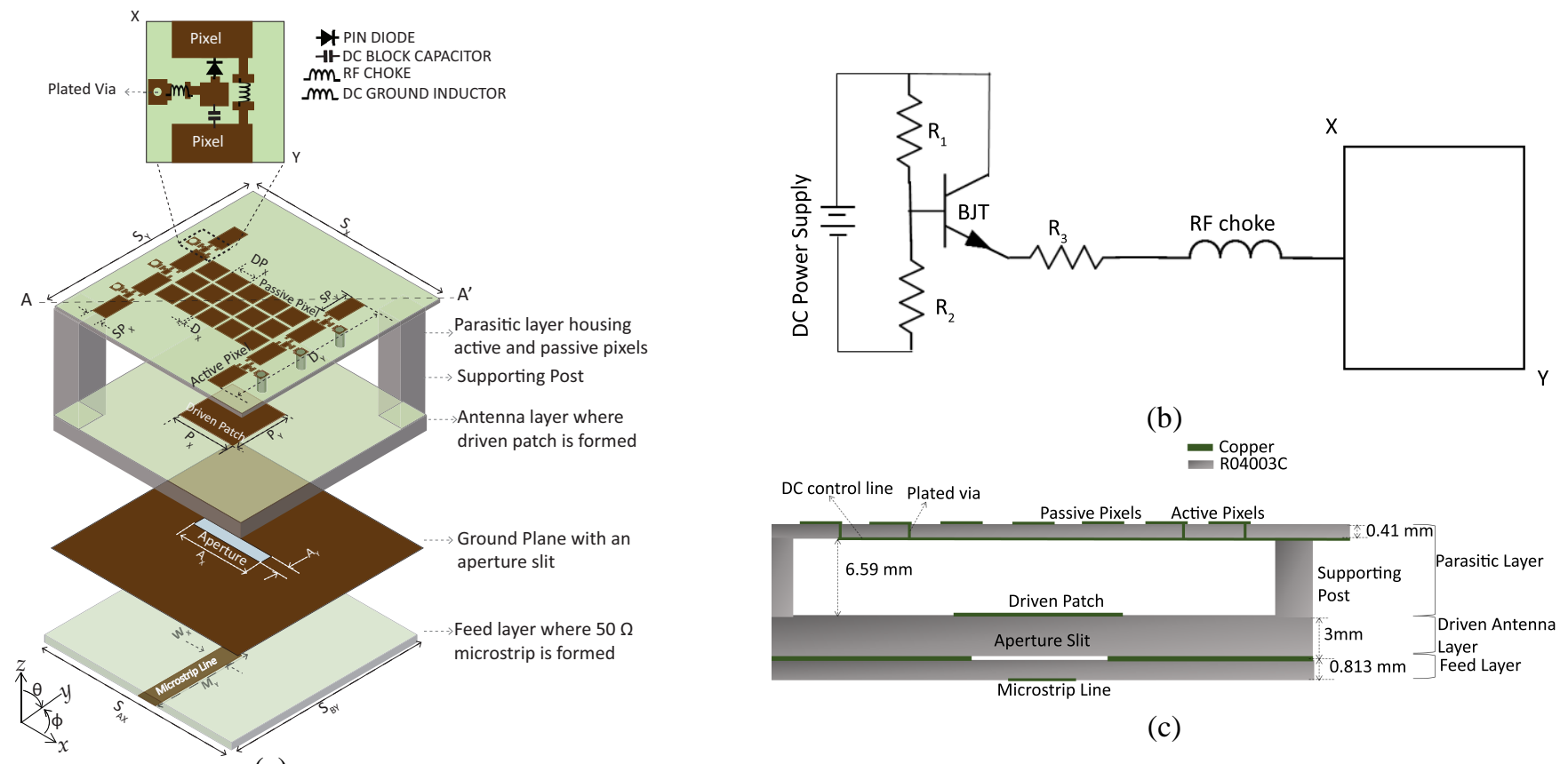

(a)

(c)

Fig. 1: (a) 3D exploded view of the RA, (b) DC biasing scheme of the PIN diode, and (c) $A A^{\prime}$ plane cross section of the RA

twelve modes of operation. In addition, this work proposes to use the RA in conjunction with a practical mode selection and user scheduling scheme that enables this RA for use in 3GPP 5G and beyond cellular access where orthogonal frequency division multiple access (OFDMA) is employed at $5 \mathrm{GHz}$ band [38], [39]. For example, system level simulations assuming $5 \mathrm{G}$ urban-micro deployment scenarios indicates that the presented RA, when used at the access points, can provide up to $29 \%$ coverage gain and $16 \%$ capacity gains at the same time. The presented RA architecture (hardware) along with its control algorithm (software) enabling to choose the optimal modes of operation (the 3-dB beam-width and beamsteering direction) with respect to the dynamically changing spatial user deployments are designed and implemented with minimal hardware and software complexities, and this is the main novelty of this work.

\section{Antenna Structure And Working Mechanism}

\section{A. Geometry of the Antenna System}

The RA of which schematics are shown in Fig. 1 consists of three main layers, namely feed, driven antenna (aperture coupled patch) and parasitic layers. Aperture coupled antenna offers a relatively broad bandwidth and a high degree of freedom in impedance tuning [40]. The radiating patch is placed on the top surface of the antenna layer, where a microstrip line with $50 \Omega$ characteristic impedance and a ground plane with an etched aperture slit are formed on the bottom and top surfaces of the feed layer, respectively. Both antenna and feed layers use Rogers R04003C substrate [41], which has been chosen for its mechanical robustness (tensile strength $=139 \mathrm{MPa}$ ), low cost and good electrical properties $\left(\epsilon_{0}=3.55, \tan \delta=0.0027\right)$. The desired band of operation has been achieved by jointly optimizing the driven patch, size and position of the aperture, the stub length (microstrip line extension beyond aperture) and substrates' thicknesses.

The parasitic layer structure, which is formed by a RO4003C substrate of $0.41 \mathrm{~mm}$ thickness supported by four vertical posts with $6.59 \mathrm{~mm}$ thickness at its four corners, is placed on top of the driven antenna layer. The main role of this structure is to provide a mechanical support for the parasitic layer, where the $6.59 \mathrm{~mm}$ air gap underneath acts as a low loss medium. The upper surface of the parasitic layer has two pixelated metallic strips where each strip comprises four equally spaced rectangular metallic pixels (active pixels). The metallic strips are placed symmetrically with respect to the center of the driven patch. A grid of $3 \times 5$ square metallic pixels (passive pixels), which are not interconnected, is placed between the two pixel strips. This pixel grid acts as PRS [33] whose surface impedance reduces the reactive component of input impedance in the driven antenna, and thereby improving the impedance matching of the RA. The multiple reflections resulting from the PRS increase the gain of the RA in the broadside direction. In terms of beam steering, the variation of current distribution over the passive pixels of the PRS surface is negligible when the active pixels are connected/disconnected by turning ON/OFF the PIN diodes. Therefore the PRS surface does not play any significant role in steering the main beam of the RA. Pixel size and inter-pixel separation have been optimized to obtain high gain and improved matching for radiation in the broadside direction.

The rectangular metallic pixels in each strip are connected via PIN diode switches, which are controlled by dc voltages applied through control lines placed on the bottom surface of the parasitic substrate. The plated vertical vias, which run through the parasitic substrate, connect the control lines on the bottom surface to the PIN diode switches on the top surface. The typical biasing mechanism of the RAs has been 
explained in details in our previous works [4], [30]. As shown in the magnified section of Fig.1(a), a DC grounding inductor has been placed between each pair of adjacent pixels to provide a common DC ground for the interconnecting PIN diode. To ensure proper RF and DC isolation, a DC blocking capacitor has been used between the PIN diode and the DC grounded pixel. RF chokes have also been used in the bias lines to minimize RF current and hence undesired mutual coupling effects of the bias lines with the radiating element. The complete bias circuit for the PIN diode is shown in Fig. 1(b), which was designed to provide a constant $10 \mathrm{~mA}$ current to the PIN diode at $1.3 \mathrm{~V}$ while in $\mathrm{ON}$ state.

An electromagnetic(EM) full-wave analysis tool [42] was used to determine the geometrical dimensions of the overall structure and surface mount device (SMD) component values resulting in the desired beam-steering and beamwidth variability capabilities. The critical geometrical dimensions and the manufacturer details of the SMD components are provided in Tables I and II.

TABLE I: Critical Design Parameters (in mm)

\begin{tabular}{|l|l|l|l|}
\hline Variable & Value & Variable & Value \\
\hline$\left(S_{X}, S_{Y}\right)$ & $(60,67)$ & $\left(P_{X}, P_{Y}\right)$ & $(12.8,12.8)$ \\
\hline$\left(S P_{X}, S P_{Y}\right)$ & $(4,8)$ & $D P_{X}$ & 6 \\
\hline$\left(D_{X}, D_{Y}\right)$ & $(1,42)$ & $W_{X}$ & 1.82 \\
\hline$\left(A_{X}, A_{Y}\right)$ & $(10,0.8)$ & $\left(S_{A X}, S_{B Y}\right)$ & $(60,60)$ \\
\hline$M_{Y}$ & 6.5 & & \\
\hline
\end{tabular}

TABLE II: Capacitance/inductance/resistance values and self resonant frequencies (SRFs) of the lumped components used in the RA

\begin{tabular}{|l|l|l|l|}
\hline Component Type & Model & Value & SRF \\
\hline PIN Diode & MA4AGFCP910 & N/A & N/A \\
\hline RF Choke & LQW15AN15NGOOD & $15 \mathrm{nH}$ & $5 \mathrm{GHz}$ \\
\hline DC grounding inductor & LQW15AN39NHOOD & $39 \mathrm{nH}$ & $5 \mathrm{GHz}$ \\
\hline DC block capacitor & GQM1885C2A1R6BB01D & $1.3 \mathrm{pF}$ & $5 \mathrm{GHz}$ \\
\hline $\begin{array}{l}\text { Bipolar Junction Tran- } \\
\text { sistor (BJT) }\end{array}$ & 2N3904 & N/A & N/A \\
\hline Resistor $\left(R_{1}\right)$ & N/A & 9.83 & N/A \\
& & $k \Omega$ & \\
\hline Resistor $\left(R_{2}\right)$ & N/A & 50.7 & N/A \\
& & $38 \Omega$ & \\
\hline Resistor $\left(R_{3}\right)$ & N/A & N/A \\
\hline
\end{tabular}

\section{B. Working Mechanism}

The working mechanism of the RA can be explained based on the classical Yagi-Uda principle [43]-[45], where the EM energy is coupled from driven element through space into parasitic dipoles resulting in a directional beam. A microstrip Yagi antenna capable of tilting its main beam away from broadside direction, where a driven patch antenna and parasitically coupled reflector and director patches are all placed in the same horizontal plane was developed [46].

In the presented RA, a driven patch antenna is mutually coupled with two reconfigurable pixelated parasitic strips that are placed above the driven element with vertical distance between driven antenna layer and parasitic layer being $0.12 \lambda$ at $5 \mathrm{GHz}$. Since a patch antenna radiates primarily in its broadside direction, this configuration increases energy coupling with the parasitic elements. The effective electrical lengths of the parasitic strip elements are changed by switching ON/OFF the interconnecting PIN diodes, making them to act either reflector or director, thus beam-steering is obtained. Similar to the case of wire antennas, a parasitic patch element with slightly longer length compared to the resonant element acts as a reflector while that with slightly shorter length acts as director [46]. The parasitic element acting as director or reflector presents capacitive or inductive effect, respectively, which changes the phases of the surface current densities on the parasitic and driven elements. This phase difference causes the main beam of the RA to be steered away from its broadside direction.

Based on the above basic EM principles, the total far-field radiation pattern of the RA structure shown in Fig. 2(a) can be expressed as [47],

$$
\begin{aligned}
F_{a}(\theta)= & F_{1}(\theta)+\frac{I_{2}}{I_{1}} F_{2}(\theta) e^{-j k_{0} d \sin (\theta)\left[1-\frac{h \cot (\theta)}{d}\right]}+ \\
& \frac{I_{3}}{I_{1}} F_{3}(\theta) e^{-j k_{0} d \sin (\theta)\left[1+\frac{h \cot (\theta)}{d}\right]}
\end{aligned}
$$

In eqn. (1), $I_{n}, n=1,2$ and 3 , is the complex current value for element $n$ and $k_{0}$ is the free space phase constant at $5 \mathrm{GHz}$. The lateral distance between driven and each parasitic element is $d=20 \mathrm{~mm}$ and vertical separation between the driven antenna and parasitic layers is $h=7 \mathrm{~mm}$. The field pattern of the RA elements in isolation can be approximated using the radiation pattern of a typical patch antenna [48], where $F_{i}(\theta)$ for $i=$ $1,2,3$ are given as follows,

$$
\begin{array}{r}
F_{1}(\theta)=\cos (\theta) \frac{\sin \left[\frac{k_{0} W_{d}}{2} \sin (\theta)\right]}{\frac{k_{0} W_{d}}{2} \sin (\theta)} \\
F_{2}(\theta)=F_{3}(\theta)=\cos (\theta) \frac{\sin \left[\frac{k_{0} W_{p}}{2} \sin (\theta)\right]}{\frac{k_{0} W_{p}}{2} \sin (\theta)}
\end{array}
$$

In eqn. (2) and (3), The widths of the driven patch and parasitic element are $W_{d}=13 \mathrm{~mm}$ and $W_{p}=6 \mathrm{~mm}$, respectively. The design parameters $W_{d}, W_{p}, d$ and $h$ are jointly optimized to perform effective beam-steering in the targeted $5 \mathrm{GHz}$ band. The range for $\theta$ angle is, $0^{\circ}<\theta<180^{\circ}$.

The complex currents, $I_{n}, n=1,2$ and 3 are calculated by integrating the magnetic fields $H_{1}, H_{2}$ and $H_{3}$ along the closed curves $C_{1}, C_{2}$ and $C_{3}$ containing element surfaces as shown in Fig. 2(a). Calculated current ratios for obtaining the radiation patterns, as shown in Figs. 2(b) and 2(c), corresponding to beam-steering and different beam-widths are given below, respectively.

$$
\begin{aligned}
& \frac{I_{2}}{I_{1}}=0.8 e^{-j 1.7} \text { and }\left|\frac{I_{3}}{I_{1}}\right| \approx 0 \text { for beam steering } \\
& \frac{I_{2}}{I_{1}}=\frac{I_{3}}{I_{1}}=0.8 e^{-j 1.7} \text { for beamwidth variability }
\end{aligned}
$$

Figs. 2(b) and 2(c) show that the approximate analytical approach for beam-steering and beam width variability agree reasonably well with full-wave analysis results.

Let us use eqn. (1) and first describe the principle of beamsteering in $x-z$ plane, where the beam-steering in all other planes can be easily explained based on this. As depicted in Fig. 2(a), when the switches $S_{1}, S_{2}$ and $S_{3}$ are turned ON, and $S_{4}, S_{5}$ and $S_{6}$ are turned OFF, the effective electrical length of the element- 2 becomes larger and that of element- 


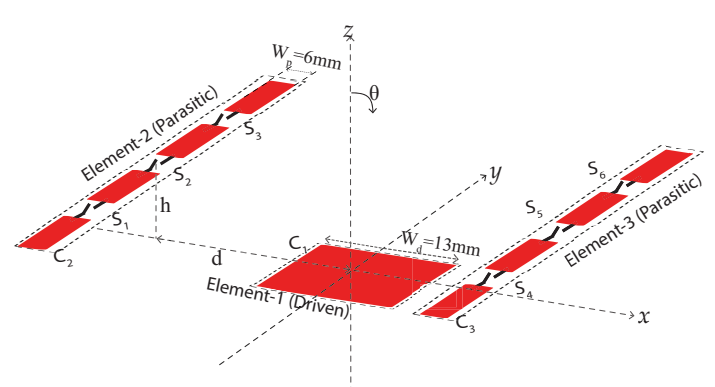

(a)

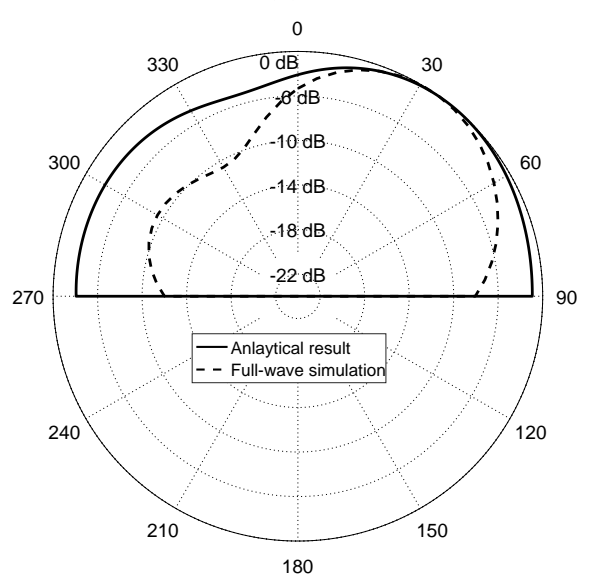

(b)

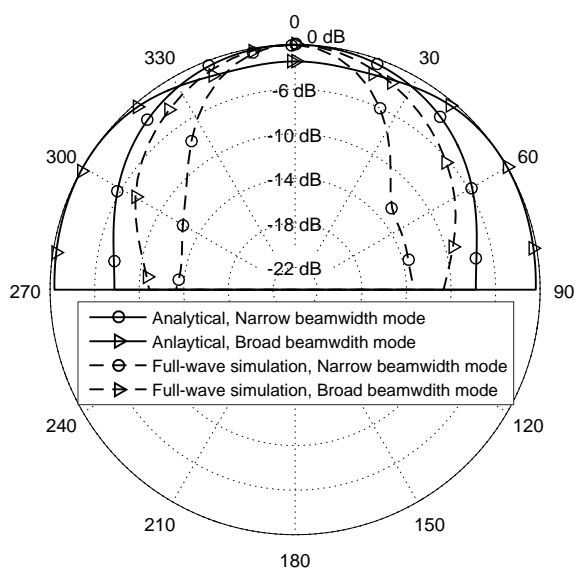

(c)

Fig. 2: (a) RA structure consisting of driven and parasitic elements. $C_{i}$ denotes the curve enclosing element- $i$, for $i=$ $1,2,3 . S_{i}$ for $i=1,2 \ldots 6$ denotes the interconnecting RF switches (b) Field patterns corresponding to beam steering in $x-z$ plane obtained by analytic method and full-wave simulation ( $S_{1}, S_{2}, S_{3}$ are ON \& $S_{4}, S_{5}, S_{6}$ are OFF) and (c) Field patterns for beam width variability in $y-z$ plane obtained by analytic method and full-wave simulation

3 becomes smaller than the length of the driven element-1. Induced currents in the parasitic elements are proportional to the effective electrical lengths and hence $I_{2}>>I_{3}$. The individual size of a parasitic pixel has been optimized such that $I_{3} \approx 0$. So, in this case, Eqn. (1) can be simplified as

$$
F_{a}(\theta)=F_{1}(\theta)+\frac{I_{2}}{I_{1}} F_{2}(\theta) e^{-j k_{0} d \sin (\theta)\left[1-\frac{h \cot (\theta)}{d}\right]}
$$

Eqn. (4) represents the total field pattern of a two-element linear array with individual element patterns of $F_{1}(\theta)$ and $F_{2}(\theta)$ and excitation currents of $I_{1}$ and $I_{2}$. Also notice that the array axis, on which the centers of the elements are placed, is the $x$-axis. The excitation currents $I_{1}$ and $I_{2}$ are complex numbers, where their ratio can be expressed as,

$$
\frac{I_{2}}{I_{1}}=X e^{-j \gamma}
$$

here $X$ is the magnitude and $\gamma$ is the phase of the current ratio. By substituting eqn. (5) into (4), we obtain the following:

$$
F_{a}(\theta)=F_{1}(\theta)+X F_{2}(\theta) e^{-j k_{0} d \sin (\theta)\left[1-\frac{h \cot (\theta)}{d}\right]-j \gamma}
$$

While $F_{1}$ and $F_{2}$ are not identical, they have been obtained from same equation (see eqns. (2) and (3) ). Therefore, they have similar patterns, which can be taken to be approximately same, $F(\theta)=F_{1}(\theta) \approx F_{2}(\theta)$, for the ease of analysis. The parasitic elements have been optimized such that $\left|I_{1}\right| \approx\left|I_{2}\right|$ and hence $|X| \approx 1$. Now, (6) can further be simplified as

$$
F_{a}(\theta)=F(\theta)\left(1+e^{-j k_{0} d \sin (\theta)\left[1-\frac{h \cot (\theta)}{d}\right]-j \gamma}\right)
$$

It can be readily observed that $\left(1+e^{-j k_{0} d \sin (\theta)\left[1-\frac{h \cot (\theta)}{d}\right]-j \gamma}\right)$ in eqn. (7) is the array factor of a two-element linear array consisting of element-2 (parasitic) and element-1 (driven antenna) of which centers lie on the $x$-axis, which is the array axis. The beam steering is performed in the plane that contains and is orthogonal to the array axis, which is the $x-z$ plane. In eqn. (7), $\gamma$ is the phase gradient which controls the steering angle of the main beam of the array, which in this case is the RA. With this analysis, it can be readily seen that while turning $S_{1}, S_{2}$ and $S_{3} \mathrm{ON}$, and keeping $S_{4}, S_{5}$ and $S_{6}$ in OFF state steers the beam toward $+x$ direction, keeping $S_{1}, S_{2}$ and $S_{3}$ in OFF state, and turning $S_{4}, S_{5}$ and $S_{6}$ ON steers the beam toward $-x$ direction. The corresponding representative radiation patterns for these cases are shown in Figs. 4(a) and 4(b).

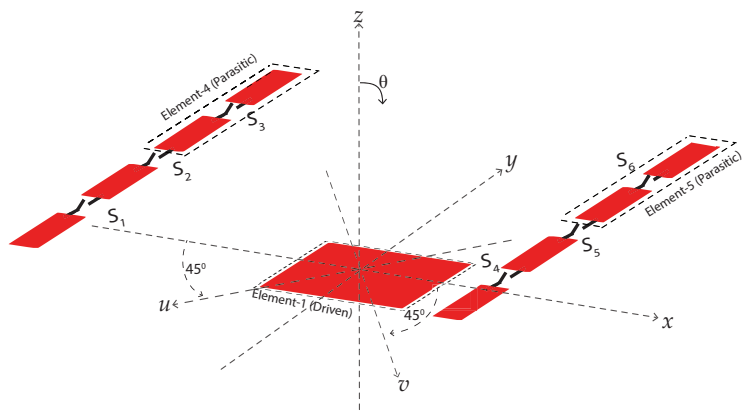

Fig. 3: RA configurations for beam steering in $y-z$ plane

By using eqns. (1)-(7), one can see that the beam-steering along $+u$ and $-u$ axes are achieved, when the centers of array 


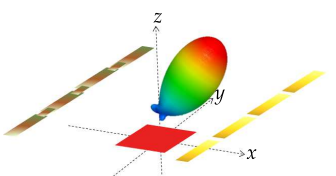

(a)

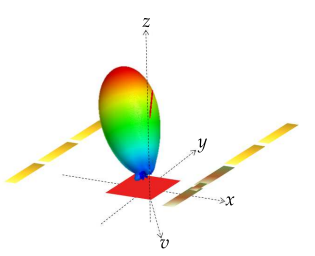

(f)

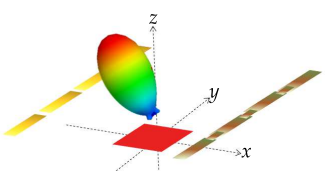

(b)

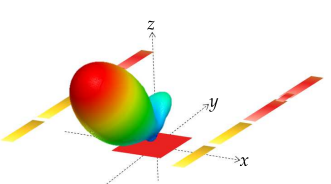

(g)

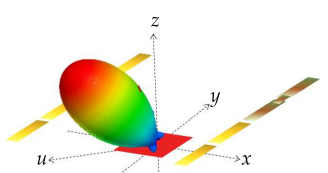

(c)

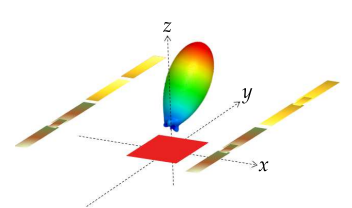

(h)

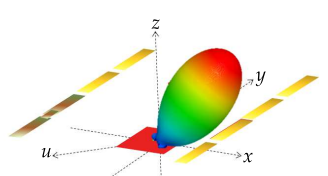

(d)

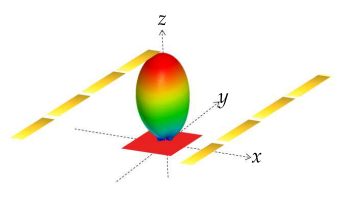

(i)

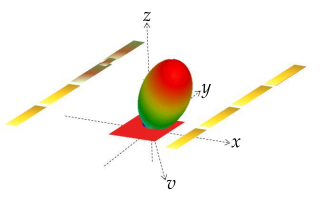

(e)

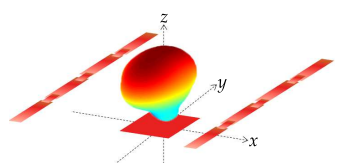

(j)

Fig. 4: Representative radiation patterns in $\mathrm{dB}$ scale for beam steering toward (a) $+x$ (b) $-x$ (c) $+u$ (d) $-u$ (e) $+v$ (f) $-v$ (g) $-y$ and $(\mathrm{h})+y$ direction, and (i) narrow and (j) broad beamwidth. Yellow colored pixels are disconnected and red colored pixels are connected.

elements lie on the $u$-axis, i.e., the array axis is $u$-axis. For beam-steering toward $+u$ direction, switch $S_{6}$ is turned $\mathrm{ON}$ and all other switches are turned OFF, and for beamsteering toward $-u$ direction, switch $S_{1}$ is turned $\mathrm{ON}$ and all other switches are turned OFF. The corresponding patterns are shown in Figs. 4(c) and 4(d). In an analogous way, the beamsteering along $v$-axis, i.e., toward $+v$ and $-v$ directions, can be explained, where the corresponding patterns are as shown in Figs. 4(e) and 4(f).

The beam steering along the $y$-axis can be achieved by creating an array geometry where the parasitic elements and the driven element are aligned along both the $u$-axis and $v$-axis (see Fig. 3). In this case, the resultant field pattern becomes the vector summation of the patterns along $u$-axis and $v$-axis, thus one can obtain a steered-beam pattern along $y$-axis. This can be accomplished in two ways: (1) Switches $S_{3}$ and $S_{6}$ are turned $\mathrm{ON}$ and all other switches are kept in the OFF state, thereby element-4 parasitic and element- 1 driven patch are aligned along $v$-axis, and element-5 parasitic and element- 1 driven patch are aligned along $u$-axis. This configuration results in beam steering towards $-y$ direction, and (2) Switches $S_{1}$ and $S_{4}$ are turned ON and all other switches are kept in the OFF state, thereby beam is steered towards $+y$ direction.The corresponding patterns are shown in Figs. 4(g) and $4(\mathrm{~h})$.

The 3-dB beamwidth of the presented RA can be broadened in $y-z$ plane by turning all switches ON. In this case, the induced current magnitudes in both elements- 2 and 3 become close $\left(\left|\frac{I_{2}}{I_{1}}\right|=\left|\frac{I_{3}}{I_{1}}\right|=0.8\right)$ to element-1. Elements-1,-2 and -3 form a linear array along $x$-axis, as shown in Fig. 2(a). Due to the phase differences between the element currents, resultant radiation pattern using eqn. (1) can be shown to broaden the 3 - $\mathrm{dB}$ beamwidth in $y-z$ plane compared to the case where all switches are in OFF state corresponding to a patch pattern with a narrow beamwidth. The corresponding representative radiation patterns for narrow and broad beam widths are shown in Figs. 4(i) and 4(j), respectively.

\section{PROTOTYPE CHARACTERIZATION AND NUMERICAL EXAMPLES}

\section{A. Measurement And Simulation Results}

A prototype RA was fabricated based on standard printed circuit board fabrication processes and measured to validate the theoretical analyses and simulated results. Fig. 5 shows the photograph of the RA measurement setup where the six PIN diode switches interconnecting the adjacent pixels are numbered in order to identify the switch status for each mode of operation.The switch configurations and associated modes of operation are shown in Table III.

TABLE III: Switch Configuration for desired modes of operation $(1=\mathrm{ON}, 0=\mathrm{OFF},(\theta, \phi)$ indicate peak gain angles)

\begin{tabular}{|c|c|c|c|c|c|c|c|c|l|}
\hline Modes & $\theta$ & $\theta_{3 d B}$ & $\phi$ & $S_{1}$ & $S_{2}$ & $S_{3}$ & $S_{4}$ & $S_{5}$ & $S_{6}$ \\
\hline 1 & $0^{\circ}$ & $40^{\circ}$ & $0^{\circ}$ & 0 & 0 & 0 & 0 & 0 & 0 \\
\hline 2 & $40^{\circ}$ & & $0^{\circ}$ & 0 & 0 & 0 & 1 & 1 & 1 \\
\hline 3 & $-40^{\circ}$ & & $0^{\circ}$ & 1 & 1 & 1 & 0 & 0 & 0 \\
\hline 4 & $40^{\circ}$ & & $45^{\circ}$ & 0 & 0 & 0 & 0 & 0 & 1 \\
\hline 5 & $-40^{\circ}$ & & $45^{\circ}$ & 1 & 0 & 0 & 0 & 0 & 0 \\
\hline 6 & $30^{\circ}$ & & $90^{\circ}$ & 0 & 0 & 1 & 0 & 0 & 1 \\
\hline 7 & $-30^{\circ}$ & & $90^{\circ}$ & 1 & 0 & 0 & 1 & 0 & 0 \\
\hline 8 & $40^{\circ}$ & & $-45^{\circ}$ & 0 & 0 & 1 & 0 & 0 & 0 \\
\hline 9 & $-40^{\circ}$ & & $-45^{\circ}$ & 0 & 0 & 0 & 1 & 0 & 0 \\
\hline 10 & $0^{\circ}$ & $100^{\circ}$ & $45^{\circ}$ & 1 & 0 & 0 & 0 & 0 & 1 \\
\hline 11 & $0^{\circ}$ & $100^{\circ}$ & $90^{\circ}$ & 1 & 1 & 1 & 1 & 1 & 1 \\
\hline 12 & $0^{\circ}$ & $100^{\circ}$ & $-45^{\circ}$ & 0 & 0 & 1 & 1 & 0 & 0 \\
\hline
\end{tabular}

The measurements included the reflection coefficients and realized gain patterns, which are cut at $5 \mathrm{GHz}$, for all twelve modes of operations. For the gain patterns, only the co-pol components are plotted as the cross-pol components are at least $-10 \mathrm{~dB}$ less than the co-pol components. The simulated and measured results for modes 1-9 are shown in Figs. 6, $7,8,9$ and 10 with good agreement between simulations and measurements. For all these modes, the common bandwidth covers 4.9-5.1 GHz band, and the maximum realized gain of $\sim 8 \mathrm{~dB}$ is achieved. Fig. 11(a) shows the variations of simulated realized gains at the associated steered angles $\left(\theta=-40^{\circ}\right.$ and $40^{\circ}$, for modes 2 and 3 , respectively) with respect to 


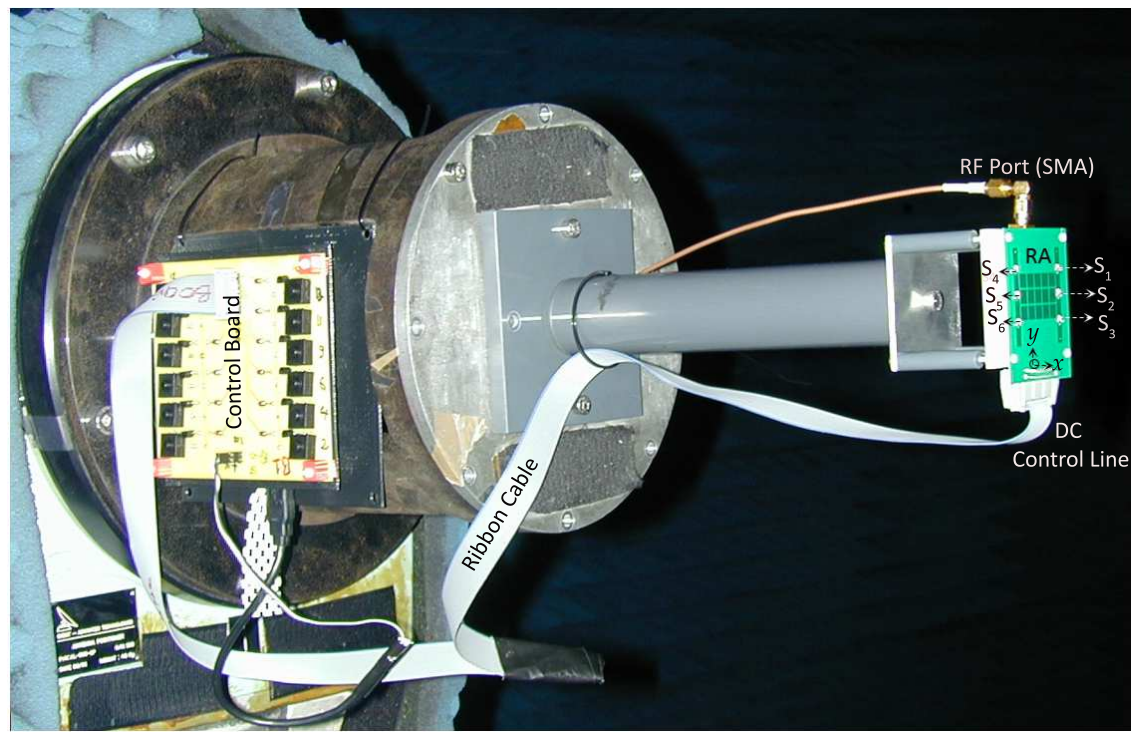

Fig. 5: Photograph of the RA measurement setup showing numbered inter-pixel PIN diode switches

frequency. These results demonstrate that the steered angles are maintained and gains stay reasonably flat over the entire frequency band for both modes. In Fig. 11(b), simulated axial ratios with respect to frequency are shown for modes 2 and 3, indicating that the RA maintains linear polarization over the entire $4.9-5.1 \mathrm{GHz}$ band. Other modes show similar behavior in terms of gain flatness and polarization purity. Fig.11(c) shows the effect of the parasitic layer on the coand cross-pol patterns of the RA. While the cross-pol pattern remains relatively unchanged $(<-20 \mathrm{~dB})$, the small reduction in the co-pol component can be attributed to the RF losses of the PIN diodes and the bias circuitry. The beamwidth variability modes, i.e., modes 10-12, are shown in Figs. 12, 13 and 14, again with good agreement between simulation and measurement results. From these figures, an average of $60^{\circ}$ increase in the $3-\mathrm{dB}$ beamwidth in $\phi=45^{\circ}, 90^{\circ}$ and, $-45^{\circ}$ planes as compared to the beamwidth of mode 1 (standard patch mode) can be observed.

The efficiency of the RA has been measured, where the efficiency with parasitic layer reduces to $81 \%$ compared to 95\% without the parasitic layer, which corresponds to the efficiency of the driven patch antenna. This reduction can be attributed to the RF losses of the PIN diodes, associated bias circuitry and the parasitic substrate.

\section{B. Performance of Proposed RA in OFDMA System}

The availability of multiple radiation patterns with an RA results in different signal strength coverages complicating its utilization in cellular networks. Here, we develop a method to utilize the proposed RA for a 5G cellular communication system employing OFDMA-based transmission. With OFDMA, selection of the RA mode (i.e., radiation pattern) in conjunction with user scheduling is critical to cultivate the frequency selective scheduling gains along with the spatial diversity provided by the RA mode optimization.

1) System Model: Assume a wireless network of $N$ access points (APs) each with $S$ sectors amounting to a total of $N S$ cells. We assume that each cell is equipped with the RA that can create $M$ different radiation patterns whose complex field is $\vec{B}_{j}(\theta, \phi ; \mu)$ with $\mu \in\{1, \ldots, M\}$ denoting the index of the RA mode at cell $-j$. The users are randomly distributed within the cell's coverage area and each user is equipped with a standard half-wavelength dipole antenna. Let us investigate a single-cell serving $L$ users, $U_{l}, l=1, \ldots, L$. Let $h_{l}\left(n, k ; \mu_{n}\right)$ represent the channel gain from AP RA (with mode- $\mu_{n}$ ) to the $U_{l}$ at time $t_{n}$ and frequency $f_{k}$.

We consider the OFDMA transmission scheme described in 3GPP TS 38.211 [49] with a subcarrier spacing of $\Delta_{f}=15$ KHz. For resource block- $b$ (RB-b) in a SF (consisting of $N_{s c}$ subcarriers and with a duration of $T_{S F}$ secs), the instantaneous achievable throughput (using the Shannon capacity formula) can be obtained for $U_{l}$ as ${ }^{1}$

$$
\begin{aligned}
r_{l}(b ; \mu)= & (1-\delta) N_{s c} T_{S F} \Delta_{f} \times \\
& \frac{1}{|\mathcal{D}|} \sum_{(k, n) \in \mathcal{D}} \log _{2}\left(1+\frac{P_{j}\left\|h_{l}\left(n, k ; \mu_{n}\right)\right\|^{2}}{\sigma_{k, n}^{2}}\right)
\end{aligned}
$$

where $\sigma_{k, n}^{2}$ is the interference plus noise power at subcarrier $(k, n), P_{j}$ is the transmit power at serving cell, and $\delta=|\mathcal{P}| /|\mathcal{D}|$ with $\mathcal{P}$ and $\mathcal{D}$ denoting the set of pilot and data subcarriers in RB- $b$, respectively. For a fixed RA mode $\mu$, the proportionally fair (PF) scheduler determines the user index $l^{*}$ at RB- $b$ according to

$$
l^{*}(b ; \mu)=\underset{l \mid b, \mu}{\arg \max } \frac{\hat{r}_{l}(b ; \mu)}{\bar{R}_{l}}
$$

where $\hat{r}_{l}(b ; \mu)$ is instantaneous achievable throughput estimated based on the pilot subcarriers in the resource block, and $\bar{R}_{l}$ is the average throughput. Note that with a fixed RA mode at the access point, the PF scheduler maximizes the sum of logarithms of the rates of the users corresponding to that mode.

2) Mode-selection and Scheduling: Each RA mode creates a different signal-to-noise ratio distribution, and thus, results

\footnotetext{
${ }^{1}$ For brevity, we drop the SF time index.
} 


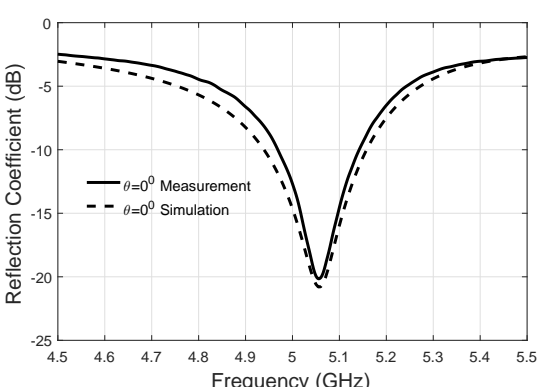

(a)

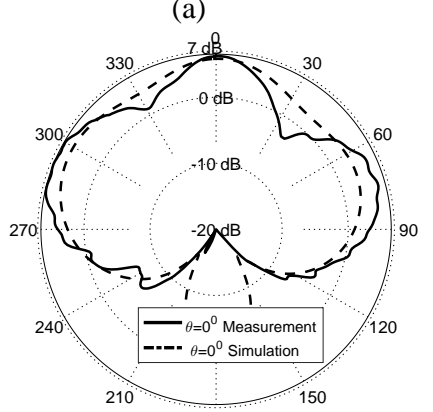

(b)

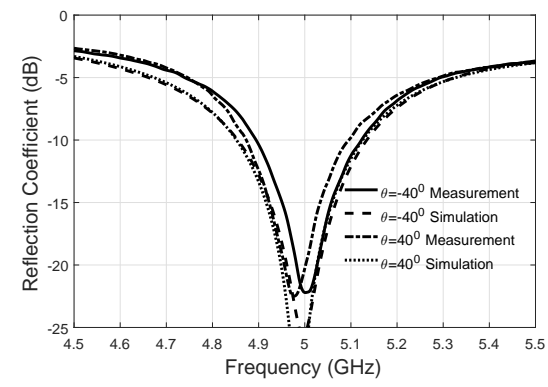

(a)

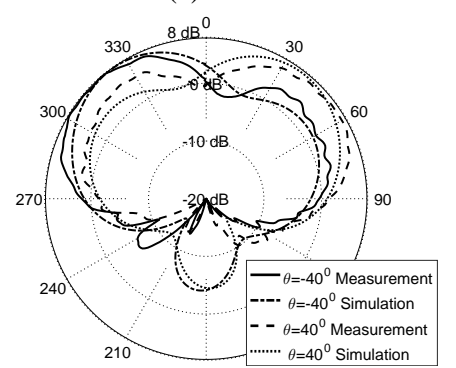

(b)

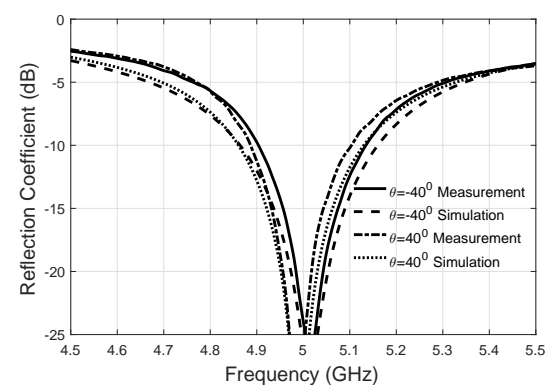

(a)

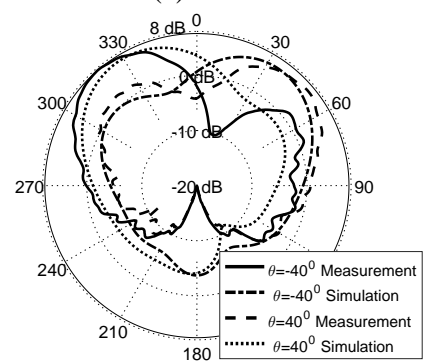

(b)

Fig. 7: Simulated and measured (a) Fig. 8: Simulated and measured (a)

Fig. 6: Simulated and measured (a) reflection coefficients, and (b) realized reflection coefficients, and (b) realized reflection coefficients, and (b) realized gain patterns of the RA for $\theta=40^{\circ}$ gain patterns of the RA for $\theta=40^{\circ}$ gain patterns of the RA for $\theta=0^{0}$ on and $\theta=-40^{0}$ on $\phi=0^{0}$ plane and $\theta=-40^{0}$ on $\phi=45^{0}$ plane $\phi=0^{0}$ plane (mode 1$)$

(modes $2 \& 3$ )

(modes 4 \& 5)

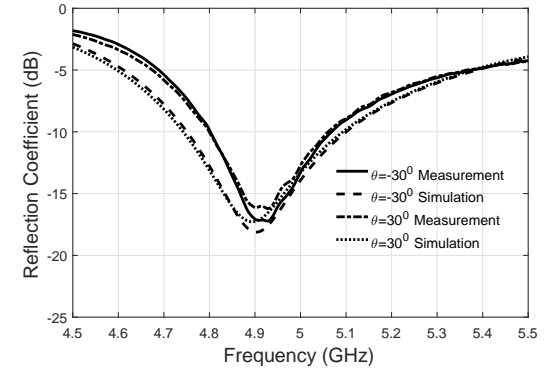

(a)

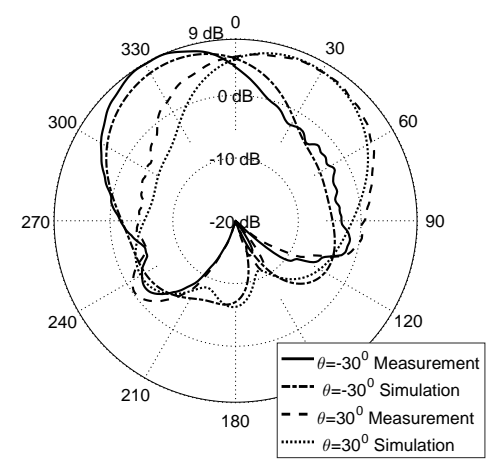

(b)

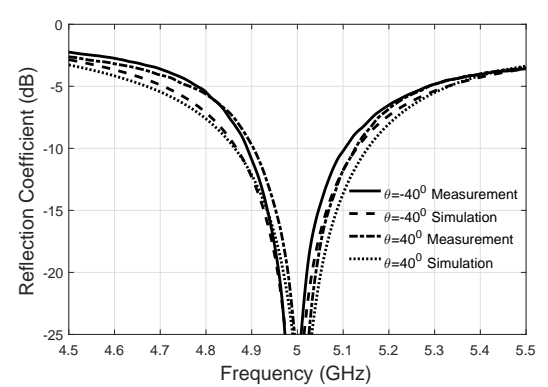

(a)

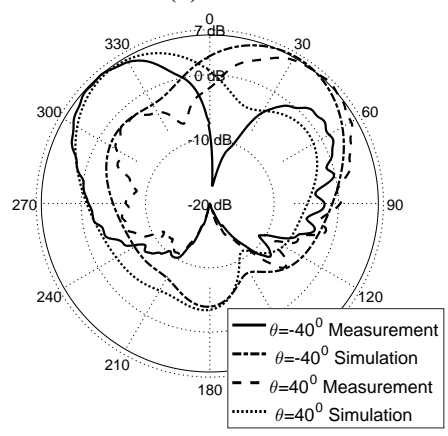

(b)

Fig. 10: Simulated and measured (a) reflection coefFig. 9: Simulated and measured (a) reflection coefficients, ficients, and (b) realized gain patterns of the RA for and (b) realized gain patterns of the RA for $\theta=30^{\circ}$ and $\theta=40^{\circ}$ and $\theta=-40^{\circ}$ on $\phi=-45^{0}$ plane (modes $\theta=-30^{\circ}$ on $\phi=90^{0}$ plane (modes $6 \& 7$ ) $8 \& 9)$

in different long-term rates for each user. This complicates an exact derivation of the PF scheduler. Here, we develop a mode-aware modified PF scheduler that imposes a constraint on the set of users enrolling in the PF prioritization. For each subframe, the AP estimates a weighted sum-rate for each mode assuming PF scheduling, and then selects the mode $\left(\mu^{*}\right)$ 


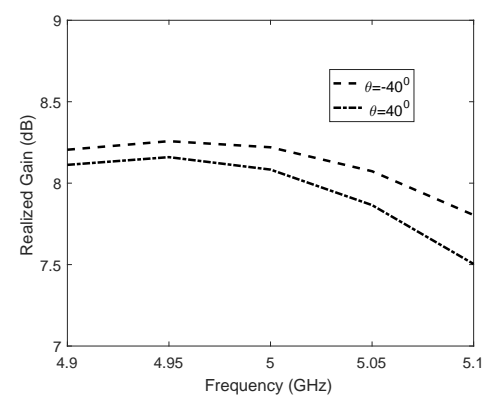

(a)

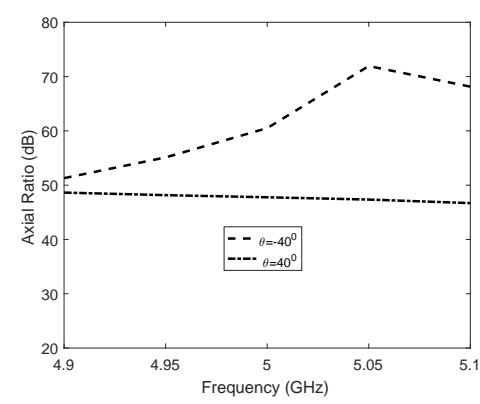

(b)

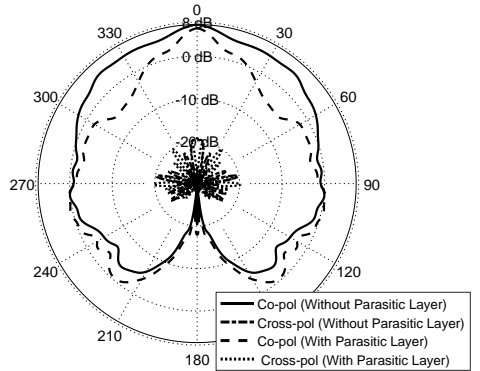

(c)

Fig. 11: For $\theta=40^{\circ}$ and $\theta=-40^{\circ}$ on $\phi=0^{0}$ plane (modes 2 and 3) (a) Realized gain variation, and (b) Axial ratio over frequency, and (c) Measured Co- and Cross-pol patterns of the RA in $y-z$ plane for mode 1 at $5 \mathrm{GHz}$ with and without the parasitic layer

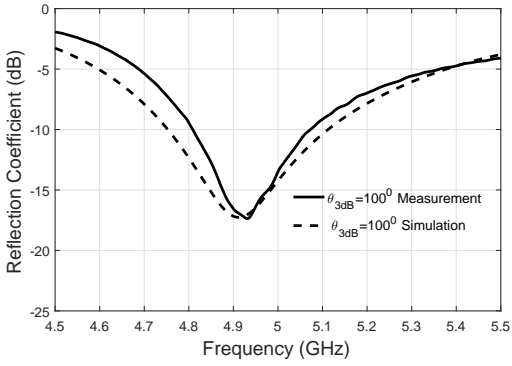

(a)

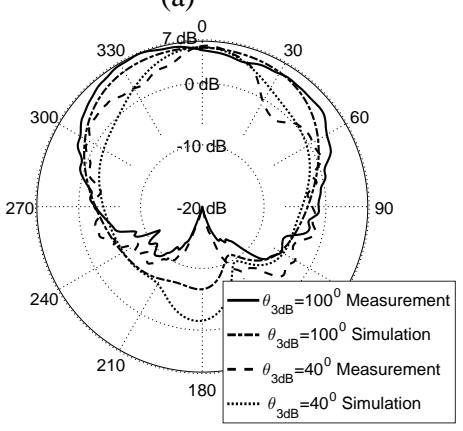

(b)

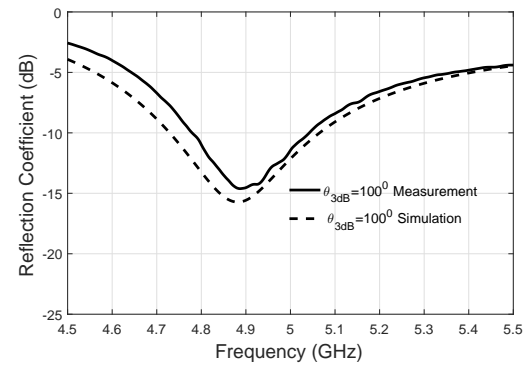

(a)

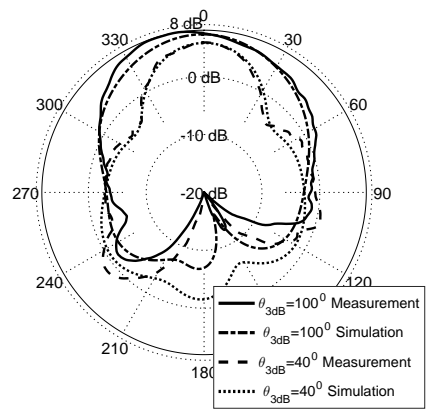

(b)

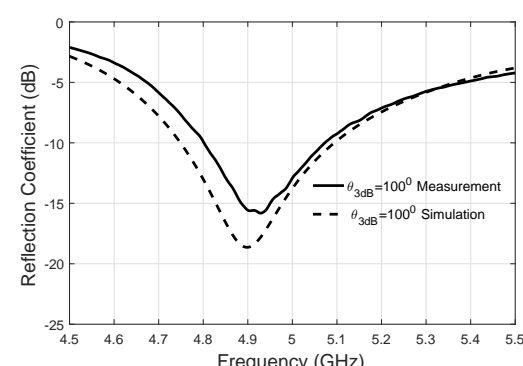

(a)

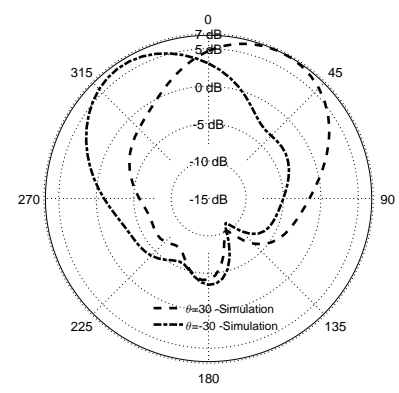

(b)

Fig. 12: Simulated and measured (a) Fig. 13: Simulated and measured (a) Fig. 14: Simulated and measured (a) reflection coefficients, and (b) realized reflection coefficients, and (b) realized reflection coefficients, and (b) realized gain patterns of the RA for $\theta_{3 d B} \in$ gain patterns of the RA for $\theta_{3 d B} \in$ gain patterns of the RA for $\theta_{3 d B} \in$ $\left\{100^{\circ}, 40^{\circ}\right\}$ on $\phi=45^{0}$ plane (mode $\left\{100^{\circ}, 40^{\circ}\right\}$ on $\phi=90^{\circ}$ plane (mode $\left\{100^{\circ}, 40^{\circ}\right\}$ on $\phi=-45^{\circ}$ plane 10)

maximizing the weighted sum-rate, i.e.,

$$
\begin{aligned}
& l^{*}\left(b ; \mu^{*}\right)= \underset{\mu \in \mathcal{M}}{\arg \max } \sum_{b} \frac{\hat{r}_{l^{*}}(b ; \mu)}{\bar{R}_{l}} \\
& \text { s. t. } l^{*}(b ; \mu)=\underset{l \in \mathcal{U}_{\mu} \mid b, \mu}{\arg \max } \frac{\hat{r}_{l}(b ; \mu)}{\bar{R}_{l}}
\end{aligned}
$$

where $\mathcal{U}_{\mu} \subseteq \mathcal{U}$ is the user subset that are allowed to be scheduled when the RA assumes mode- $\mu$. The members and size of $\mathcal{U}_{\mu}$ determine a trade-off between gains due to frequency selective scheduling and the pattern optimization. To achieve this for each mode, we propose signal strength based subset selection scheme

$$
\begin{aligned}
\mathcal{U}_{\mu} & \triangleq\left\{l: \mu \in \mathcal{M}_{l}\right\} \\
& \text { s. t. } \mathcal{M}_{l} \triangleq\left\{\mu: \frac{g_{l}(\mu)}{\max _{\mu} g_{l}(\mu)} \geq g_{t h}, l=1, \ldots, L\right\}
\end{aligned}
$$

where $g_{l}(\mu)=E_{n}\left\{\sum_{k}\left\|h_{l}(n, k ; \mu)\right\|^{2}\right\}$ is the average channel power for $U_{l}$, and $g_{t h} \in[0,1]$ is a threshold parameter controlling the cardinality of $\mathcal{U}_{\mu}$. Note that this scheme first determines the set of suitable RA modes for each user (e.g., modes that can provide acceptable signal quality to the user), and then for each mode, aggregates the users that can be served by that mode. This approach ensures that each user can be 


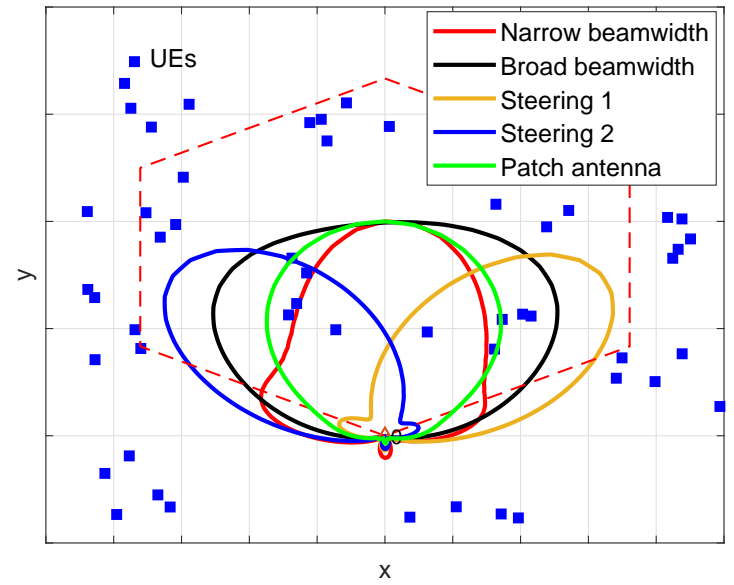

(a)

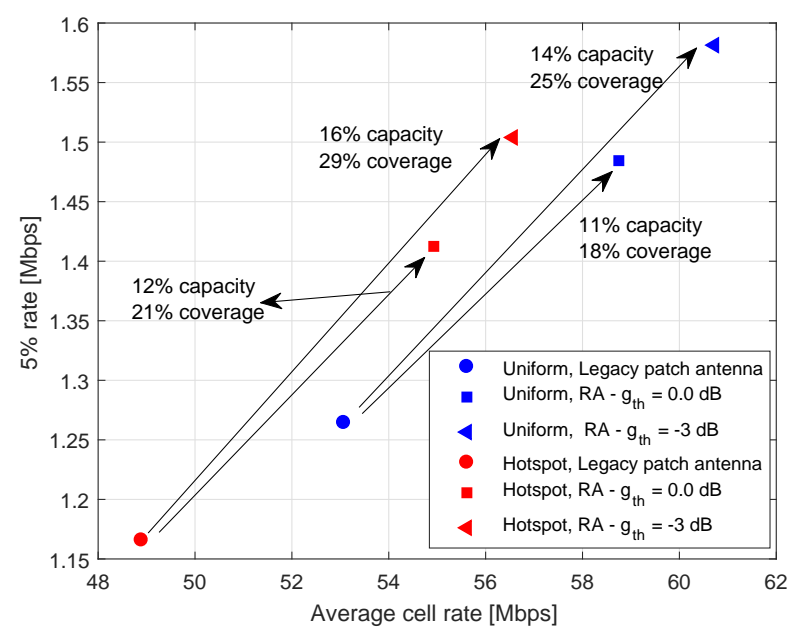

(b)

Fig. 15: (a) Radiation patterns for various RA modes and the patch antenna in a typical cell, (b) throughput-coverage performance of RA and the legacy patch antenna for two use-cases, Uniform and Hotspot.

TABLE IV: System level simulation parameters.

\begin{tabular}{|l|l|l|l|}
\hline Parameter & Value & Parameter & Value \\
\hline Number of APs & 19 & Sectors per AP & 3 \\
\hline AP-AP distance & $250 \mathrm{~m}$ & AP-UE channel & UMa [50] \\
\hline $\begin{array}{l}\text { Average number of } \\
\text { UEs per cell }\end{array}$ & 20 & UE deployment & $\begin{array}{l}\text { Uniform } \\
\text { or Hotspot }\end{array}$ \\
\hline $\begin{array}{l}\text { Average number of } \\
\text { hotspots per cell }\end{array}$ & 4 & $\begin{array}{l}\text { Number of UEs per } \\
\text { hotspot }\end{array}$ & 5 \\
\hline min AP-UE distance & $10 \mathrm{~m}$ & $\begin{array}{l}\text { min inter-cluster } \\
\text { distance }\end{array}$ & $50 \mathrm{~m}$ \\
\hline $\begin{array}{l}\text { Number of RA mo- } \\
\text { des }\end{array}$ & 12 & AP Tx Power & $30 \mathrm{dBm}$ \\
\hline Channel bandwidth & $10 \mathrm{MHz}$ & Number of SCs (N) & 600 \\
\hline
\end{tabular}

served using at least one RA mode when $g_{t h}>0$, and by any of the modes when $g_{t h}=0$.

3) Numerical Examples: System level simulation parameters for the network are provided in Table IV. Two spatial user deployments are studied: (1) Uniform user density where users are dropped uniformly randomly within the coverage area, and (2) hotspot deployment where users are dropped within $20 \mathrm{~m}$ of cluster centres that are spatially randomly distributed.

Performance is analyzed for 3 cases: APs employ (1) legacy patch antennas, (2) RAs where UEs report the strongest mode, and (3) RAs where UEs report modes having signal strength within $3 \mathrm{~dB}$ of the strongest mode. Fig. 15(a) compares the radiation patterns for various RA modes and the patch antenna in a typical cell serving several UEs for the hotspot scenario. It is seen that the RA can provide an enhanced set of beam patterns that can be adapted to spatial UE or traffic distribution. Average capacity-coverage performance for the two deployment cases is illustrated in Fig. 15(b). The coverage is defined as the 5-percentile of the cumulative distribution function (CDF) of per-user rates. It is seen that for both deployment scenarios, the RA system achieves superior capacity/coverage trade-off against the system using legacy patch antennas. For example, the RA system can provide up to $29 \%$ coverage/16\% capacity improvement for hotspot scenario and up to $25 \%$ coverage $/ 14 \%$ capacity improvement for the case of uniform user distribution. The improvements are pronounced more for hotspot scenarios as beam-steering and beamwidth control can provide better signal coverage to the hotspot areas while maintaining lower interference to unintended users. The results with $g_{t h}=0$ indicates that when a user is served with the RA-mode providing the strongest signal quality (e.g., corresponding to activation of average 4 modes in each cell under the studied scenarios), the system can achieve significant improvements. In this case, the gains are mainly due to the pattern control offered by the RA. With lower $g_{t h}$ values, the gains from multi-user diversity is enhanced for each RA mode, and along with the gains from RA pattern diversity, further improvement is obtained.

A comparison between the presented RA and similar other RAs in terms of the number of modes generated, number of switches used and reconfigurable antenna properties such as beam-steering capability, beam-width variability, pattern purity, average gain, etc is given in Table V. It can be observed that the RA of this work is advantageous for achieving larger number of modes per the number of switches used. In particular, compared to [30], this RA does not only provide larger number of modes with less number of switches, greater beam-steering range and better pattern purity but also the average antenna gain, which is $8 \mathrm{~dB}$, is increased by $\sim 1.5 \mathrm{~dB}$. Notice that the largest average gain of $14.7 \mathrm{~dB}$ for work presented in [37] uses a $1 \times 2$ antenna array as its driven element, thereby having a complex driven antenna structure. While this work achieve analog beam-steering with a range of $\sim 20^{\circ}$ and small beam-width variability, the $1 \times 2$ array structure with phase shifters and the PRS with exceedingly large number of varactors and associated biasing circuitry results in very complex antenna structure and high RF losses. Another significant advantage of the RA presented in this work compared to the ones given in Table $\mathrm{V}$ is that the proposed control algorithm enables to choose the optimal modes of 
TABLE V: Comparison among various RAs

\begin{tabular}{|l|l|l|l|l|l|l|l|l|l|l|}
\hline Publications & $\begin{array}{l}\text { No. of } \\
\text { Modes }\end{array}$ & $\begin{array}{l}\text { No. of } \\
\text { Switches }\end{array}$ & $\begin{array}{l}\text { Beam } \\
\text { Steering }\end{array}$ & $\begin{array}{l}\text { Beam } \\
\text { Steering } \\
\text { Range }\end{array}$ & $\begin{array}{l}\text { Beam } \\
\text { Width } \\
\text { Variable }\end{array}$ & $\begin{array}{l}\text { Pattern } \\
\text { Purity } \\
\text { (Cross } \\
\text { Polari- } \\
\text { zation } \\
\text { Level) }\end{array}$ & $\begin{array}{l}\text { Average } \\
\text { Gain (dB) }\end{array}$ & $\begin{array}{l}\text { Efficiency } \\
\text { Frequency } \\
\text { BW } \\
\text { (GHz) }\end{array}$ & $\begin{array}{l}\text { Joint opti- } \\
\text { mization of } \\
\text { RA } \\
\text { and control } \\
\text { algorithm }\end{array}$ \\
\hline$[30]$ & 9 & 12 & Yes & $-30^{\circ}, 30^{\circ}$ & No & $<-10 \mathrm{~dB}$ & 6.5 & N/A & $2.4-2.5$ & Yes \\
\hline$[37]$ & N/A & 100 & Yes & $-10^{\circ}, 10^{\circ}$ & Yes & $-12 \mathrm{~dB}$ & 14.7 & N/A & $1.9-2.1$ & No \\
\hline$[9]$ & 4 & 8 & Yes & $-17^{\circ}, 14^{\circ}$ & Yes & $-15 \mathrm{~dB}$ & 7 & N/A & $3.32-3.62$ & No \\
\hline$[10]$ & 9 & 4 & Yes & $-22^{\circ}, 22^{\circ}$ & No & N/A & 7.5 & $76 \%$ & $2.36-2.39$ & No \\
\hline This work & 12 & 6 & Yes & $-40^{\circ}, 40^{\circ}$ & Yes & $<-20 \mathrm{~dB}$ & 8 & $81 \%$ & $4.9-5.1$ & Yes \\
\hline
\end{tabular}

operation (the 3-dB beam-width and beam-steering direction) in an efficient way with respect to the dynamically changing spatial user deployments.

\section{Conclusion And Future Work}

A new parasitic coupling based RA design that can provide beam-steering and beamwidth control on a single compact antenna platform is developed. The proposed RA can generate 9 beam-steering and 3 beamwidth variable modes. With a novel RA mode-aware PF scheduling scheme that jointly determines the RA pattern and scheduled users, the proposed RA can provide significant improvements against the system using legacy patch antennas, e.g., up to $29 \%$ coverage and $16 \%$ throughput gains for a hotspot scenario. The improvements are critical for $5 \mathrm{G}$ wireless access where extreme broadband along with ubiquitous coverage is mandated by the $5 \mathrm{G}$ requirements. The presented RA architecture (hardware) along with its control algorithm (software) enabling to choose the optimal modes of operation (the 3-dB beam-width and beamsteering direction) with respect to the dynamically changing spatial user deployments are designed and implemented with minimal hardware and software complexities. We conclude that designing RAs in conjunction with signaling schemes and network requirements with low overhead is essential to optimize overall system performance.

\section{REFERENCES}

[1] D. Rodrigo, B. Cetiner, and L. Jofre, "Frequency, radiation pattern and polarization reconfigurable antenna using a parasitic pixel layer," IEEE Transactions on Antennas and Propagation, vol. 62, no. 6, pp. 3422 3427, June 2014.

[2] Z. Li, D. Rodrigo, L. Jofre, and B. Cetiner, "A new class of antenna array with a reconfigurable element factor," IEEE Transactions on Antennas and Propagation, vol. 61, no. 4, pp. 1947-1955, April 2013.

[3] C. J. Panagamuwa, A. Chauraya, and J. C. Vardaxoglou, "Frequency and beam reconfigurable antenna using photoconducting switches," IEEE Transactions on Antennas and Propagation, vol. 54, no. 2, pp. 449454, Feb 2006.

[4] D. Rodrigo, J. Romeu, B. A. Cetiner, and L. Jofre, "Pixel reconfigurable antennas: Towards low-complexity full reconfiguration," in 2016 10th European Conference on Antennas and Propagation (EuCAP), April 2016, pp. 1-5.

[5] A. Zohur, H. Mopidevi, D. Rodrigo, M. Unlu, L. Jofre, and B. A. Cetiner, "Rf mems reconfigurable two-band antenna," IEEE Antennas and Wireless Propagation Letters, vol. 12, pp. 72-75, 2013.

[6] M. W. Young, S. Yong, and J. T. Bernhard, "A miniaturized frequency reconfigurable antenna with single bias, dual varactor tuning," IEEE Transactions on Antennas and Propagation, vol. 63, no. 3, pp. 946951, March 2015.

[7] A. J. King, J. F. Patrick, N. R. Sottos, S. R. White, G. H. Huff, and J. T. Bernhard, "Microfluidically switched frequency-reconfigurable slot antennas," IEEE Antennas and Wireless Propagation Letters, vol. 12, pp. 828-831, 2013.
[8] S. V. Hum and J. Perruisseau-Carrier, "Reconfigurable reflectarrays and array lenses for dynamic antenna beam control: A review," IEEE Transactions on Antennas and Propagation, vol. 62, no. 1, pp. 183-198, Jan 2014.

[9] W. Q. Deng, X. S. Yang, C. S. Shen, J. Zhao, and B. Z. Wang, "A dual-polarized pattern reconfigurable yagi patch antenna for microbase stations," IEEE Transactions on Antennas and Propagation, vol. 65, no. 10, pp. 5095-5102, Oct 2017.

[10] M. Jusoh, T. Aboufoul, T. Sabapathy, A. Alomainy, and M. R. Kamarudin, "Pattern-reconfigurable microstrip patch antenna with multidirectional beam for wimax application," IEEE Antennas and Wireless Propagation Letters, vol. 13, pp. 860-863, 2014.

[11] J. Hu, S. Lin, and F. Dai, "Pattern reconfigurable antenna based on morphing bistable composite laminates," IEEE Transactions on Antennas and Propagation, vol. 65, no. 5, pp. 2196-2207, May 2017.

[12] N. H. Chamok, M. H. Ylmaz, H. Arslan, and M. Ali, "High-gain pattern reconfigurable mimo antenna array for wireless handheld terminals," IEEE Transactions on Antennas and Propagation, vol. 64, no. 10, pp. 4306-4315, Oct 2016.

[13] B. Cetiner, H. Jafarkhani, J.-Y. Qian, H. J. Yoo, A. Grau, and F. De Flaviis, "Multifunctional reconfigurable MEMS integrated antennas for adaptive MIMO systems," IEEE Communications Magazine, vol. 42, no. 12, pp. 62-70, 2004.

[14] Y. Pan, Y. Hou, M. Li, R. M. Gerdes, K. Zeng, M. A. Towfiq, and B. A. Cetiner, "Message integrity protection over wireless channel: Countering signal cancellation via channel randomization," IEEE Transactions on Dependable and Secure Computing, pp. 1-1, 2017.

[15] 3GPP, "TS 22.261: Service requirements for the 5G system - Stage 1 (rel. 15)," [Online]. Available: http://www.3gpp.org/ftp//Specs/archive/ 22' series/22.261/22261-f30.zip, Dec. 2017.

[16] ETSI, "ETSI TR 138913 v14.2.0 (2017-05)," [Online]. Available: http://www.etsi.org/deliver/etsi tr/ $138900^{\circ} 138999 / 138913 / 14.02 .00^{\circ} 60 /$ tr $^{*} 138913 v 140200$ p.pdf, $\quad$ May 2017.

[17] H. Tullberg, P. Popovski, Z. Li, M. A. Uusitalo, A. Hoglund, O. Bulakci, M. Fallgren, and J. F. Monserrat, "The metis 5g system concept: Meeting the $5 \mathrm{~g}$ requirements," IEEE Communications Magazine, vol. 54, no. 12 , pp. 132-139, December 2016.

[18] ITU-R, "Report ITU-R M.2320-0: Future technology trends of terrestrial IMT systems," [Online]. Available: https://www.itu.int/dms pub/itu-r/ opb/rep/R-REP-M.2320-2014-PDF-E.pdf, Nov 2014.

[19] Z. Niu, Y. Wu, J. Gong, and Z. Yang, "Cell zooming for cost-efficient green cellular networks," IEEE Communications Magazine, vol. 48, no. 11, pp. 74-79, November 2010.

[20] C. Y. Wang, C. H. Ko, H. Y. Wei, and A. V. Vasilakos, "A voting-based femtocell downlink cell-breathing control mechanism," IEEE/ACM Transactions on Networking, vol. 24, no. 1, pp. 85-98, Feb 2016.

[21] A. Tall, Z. Altman, and E. Altman, "Virtual sectorization: Design and self-optimization," in 2015 IEEE 81st Vehicular Technology Conference (VTC Spring), May 2015, pp. 1-5.

[22] G. H. Huff, J. Feng, S. Zhang, and J. T. Bernhard, "A novel radiation pattern and frequency reconfigurable single turn square spiral microstrip antenna," IEEE Microwave and Wireless Components Letters, vol. 13, no. 2, pp. 57-59, Feb 2003.

[23] L. Petit, L. Dussopt, and J. M. Laheurte, "Mems-switched parasiticantenna array for radiation pattern diversity," IEEE Transactions on Antennas and Propagation, vol. 54, no. 9, pp. 2624-2631, Sept 2006.

[24] S. Zhang, G. H. Huff, J. Feng, and J. T. Bernhard, "A pattern reconfigurable microstrip parasitic array," IEEE Transactions on Antennas and Propagation, vol. 52, no. 10, pp. 2773-2776, Oct 2004. 
[25] X.-S. Yang, B.-Z. Wang, and Y. Zhang, "Pattern-reconfigurable quasi-yagi microstrip antenna using a photonic band gap structure," Microwave and Optical Technology Letters, vol. 42, no. 4, pp. 296-297, 2004. [Online]. Available: http://dx.doi.org/10.1002/mop.20283

[26] S. Nikolaou, G. E. Ponchak, J. Papapolymerou, and M. M. Tentzeris, "Design and development of an annular slot antenna (asa) with a reconfigurable radiation pattern," in 2005 Asia-Pacific Microwave Conference Proceedings, vol. 5, Dec 2005.

[27] X.-S. Yang, B.-Z. Wang, and W. Wu, "Pattern reconfigurable patch antenna with two orthogonal quasi-yagi arrays," in 2005 IEEE Antennas and Propagation Society International Symposium, vol. 2B, July 2005, pp. 617-620 vol. 2B.

[28] C. won Jung, M. jer Lee, G. P. Li, and F. D. Flaviis, "Reconfigurable scan-beam single-arm spiral antenna integrated with rf-mems switches," IEEE Transactions on Antennas and Propagation, vol. 54, no. 2, pp. 455-463, Feb 2006.

[29] J. C. Maloney, M. P. Kesler, L. M. Lust, L. N. Pringle, T. L. Fountain, P. H. Harms, and G. S. Smith, "Switched fragmented aperture antennas," in Antennas and Propagation Society International Symposium, 2000. IEEE, vol. 1, July 2000, pp. 310-313 vol.1.

[30] Z. Li, E. Ahmed, A. M. Eltawil, and B. A. Cetiner, "A beam-steering reconfigurable antenna for wlan applications," IEEE Transactions on Antennas and Propagation, vol. 63, no. 1, pp. 24-32, Jan 2015.

[31] Z. Li, H. Mopidevi, O. Kaynar, and B. A. Cetiner, "Beam-steering antenna based on parasitic layer," Electronics Letters, vol. 48, no. 2, pp. 59-60, January 2012.

[32] X. Yuan, Z. Li, D. Rodrigo, H. Mopidevi, O. Kaynar, L. Jofre, and B. Cetiner, "A parasitic layer-based reconfigurable antenna design by multi-objective optimization," IEEE Transactions on Antennas and Propagation, vol. 60, no. 6, pp. 2690-2701, June 2012.

[33] G. V. Trentini, "Partially reflecting sheet arrays," IRE Transactions on Antennas and Propagation, vol. 4, no. 4, pp. 666-671, October 1956.

[34] A. P. Feresidis and J. C. Vardaxoglou, "High gain planar antenna using optimised partially reflective surfaces," IEE Proceedings - Microwaves, Antennas and Propagation, vol. 148, no. 6, pp. 345-350, Dec 2001.

[35] A. R. Weily, T. S. Bird, and Y. J. Guo, "A reconfigurable high-gain partially reflecting surface antenna," IEEE Transactions on Antennas and Propagation, vol. 56, no. 11, pp. 3382-3390, 2008.

[36] T. Debogovic, J. Bartoli, and J. Perruisseau-Carrier, "Array-fed partially reflective surface antenna with dynamic beamwidth control and beamsteering," in 2012 6th European Conference on Antennas and Propagation (EUCAP), March 2012, pp. 3599-3603.

[37] T. Debogovic and J. Perruisseau-Carrier, "Array-fed partially reflective surface antenna with independent scanning and beamwidth dynamic control," IEEE Transactions on Antennas and Propagation, vol. 62, no. 1, pp. 446-449, 2014.

[38] 5GAmericas, "5G spectrum recommendations," [Online]. Available: http://www.5gamericas.org/ files/9114/9324/1786/ 5GA'5G'Spectrum Recommendations'2017'FINAL.pdf, April 2017.

[39] ITU, "RESOLUTION 239(WRC-15): Studies concerning wireless access systems including radio local area networks in the frequency bands between $5150 \mathrm{mhz}$ and 5925 mhz," [Online]. Available: http://www.itu.int/dms pub/itur/oth/0c/0a/R0C0A00000C0017PDFE.pdf, April 2015.

[40] D. M. Pozar, "Microstrip antenna aperture-coupled to a microstripline," Electronics Letters, vol. 21, no. 2, pp. 49-50, January 1985.

[41] Rogers, "Rogers corp., chandler, az," 2016.

[42] HFSS, "Ansoft corp., pittsburgh, pa," 2015.

[43] S. Uda, "Wireless beam of short electric waves," J. IEE, pp. 273-282, 1926.

[44] H. Yagi, "Beam transmission of ultra short waves," Proceedings of the Institute of Radio Engineers, vol. 16, no. 6, pp. 715-740, 1928.

[45] J. Huang, "Planar microstrip yagi array antenna," in Antennas and Propagation Society International Symposium, 1989. AP-S. Digest. IEEE, 1989, pp. 894-897.

[46] J. Huang and A. C. Densmore, "Microstrip yagi array antenna for mobile satellite vehicle application," IEEE Transactions on Antennas and Propagation, vol. 39, no. 7, pp. 1024-1030, Jul 1991.

[47] I. Rana, N. Alexopoulos, and P. Katehi, "Theory of microstrip yagi-uda arrays," Radio Science, vol. 16, no. 6, pp. 1077-1079, 1981.

[48] W. L. Stutzman and G. A. Thiele, Antenna theory and design. John Wiley \& Sons, 2012.

[49] 3GPP, "TS 38.211: NR - physical channels and modulation (rel. 15)," [Online]. Available: http://www.3gpp.org/ftp//Specs/archive/ 38'series/38.211/38211-100.zip, September 2017.
[50] '3GPP', "TR 36.873: Study on 3D channel model for LTE (Rel. 12)," [Online]. Available: http://www.3gpp.org/ftp/Specs/archive/ 36'series/36.873/36873-c20.zip, June 2015.

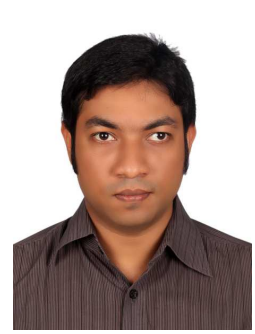

Md. Asaduzzaman Towfiq is a Ph.D. student at Utah State University, Logan, Utah. He received B.S. in electrical and electronics engineering from Bangladesh University of Engineering and Technology, Dhaka, in 2013. His research interests include reconfigurable antenna, phased array and mmWave antennas.

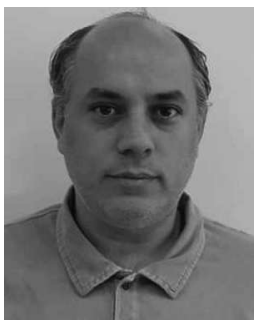

Israfil Bahceci received the B.S. degree in electrical engineering from Bilkent University, Ankara, Turkey, in 1999, the M.S. degree in electrical engineering from Arizona State University, Tempe, AZ, USA, in 2001, and the Ph.D. degree in electrical engineering from the Georgia Institute of Technology, Atlanta, GA, USA, in 2005. He is currently at Ericsson Canada, Inc. He was a Post-Doctoral Fellow with the University of Waterloo, Waterloo, ON, Canada, from 2005 to 2007. He was a Research and Development Engineer with Nortel Networks, Canada, from 2007 to 2009. He was with Huawei Technologies, Canada, from 2009 to 2011. He was with the TOBB University of Economics and Technology, Ankara, Turkey, from 2011 to 2015. He was a Research Scientist with the Department of Electrical and Computer Engineering, Utah State University, Logan, UT, USA, from 2015 to 2017. His current research interests include systems, with a particular focus on communication and signal processing, wireless and mobile communications, distributed estimation/detection, and reconfigurable antenna systems for $5 \mathrm{G}$ and beyond systems.

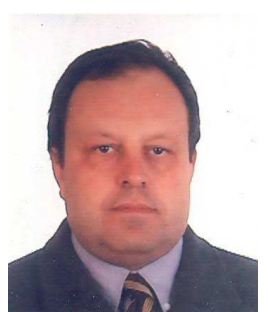

Sebastián Blanch was born in Barcelona, Spain, in 1961. He received the Ingeniero and Doctor Ingeniero degrees in Telecommunication Engineering, both from the Polytechnic University of Catalonia (UPC), Barcelona, Spain, in 1989 and 1996, respectively. In 1989, he joined the Electromagnetic and Pho-tonics Engineering Group of the Signal Theory and Communications Department. Currently, he is Associate Professor at UPC. His research interests are antenna near field measurements, antenna diagnostics, and antenna design. 


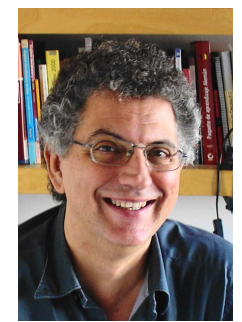

Jordi Romeu was born in Barcelona, Spain, in 1962. He received the Ingeniero de Telecomunicacin and Doctor Ingeniero de Telecomunicacon degree from the Universitat Politcnica de Catalunya (UPC), Barcelona, Spain, in 1986 and 1991, respectively. He has been with the Electromagnetic and Photonic Engineering Group, Signal Theory and Communications Department, UPC, since 1985, where he is currently a full Professor, where he is involved in the research of antenna near-eld measurements, antenna diagnostics, and antenna design. He joined the Antenna Laboratory, University of California, Los Angeles, CA, USA in 1999, as a Visiting Scholar, under the North Atlantic Treaty Organization Scientic Program Scholarship. In 2004, he joined the University of California, Irvine, CA, USA. He has authored 50 refereed papers in international journals and 50 conference proceedings and holds several patents. Dr. Romeu received the Grand Winner of the European IT Prize by the European Commission, for his contributions in the development of fractal antennas in 1998.

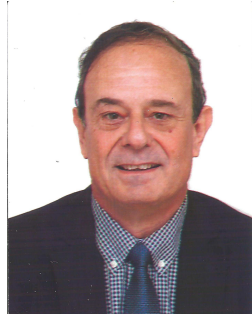

Luis Jofre was born in Canet de Mar, Spain, in 1956. He received the M.Sc. (Ing.) and Ph.D. (Doctor Eng.) degrees in electrical engineering (telecommunication engineering) from the Technical University of Catalonia (UPC), Barcelona, Spain, in 1978 and 1982, respectively. He was a Research Assistant with the Electrophysics Group, UPC, from 1979 to 1980 , where he was involved in the analysis and near-field measurement of antennas and scatterers. From 1981 to 1982, he was with the cole Suprieure dElectricit Paris, Gif-sur-Yvette, France, where he was involved in microwave antenna design and imaging techniques for medical and industrial applications. Since 1982, he has been with the Communications Department, Telecommunication Engineering School, UPC, as an Associate Professor and then as a full Professor since 1989. From 1986 to 1987, he was a Visiting Fulbright Scholar with the Georgia Institute of Technology, Atlanta, GA, USA, where he was involved in antenna nearfield measurements and electromagnetic imaging. From 1989 to 1994, he was the Director of the Telecommunication Engineering School, UPC, and from 1994 to 2000, he was the UPC Vice-Rector for academic planning. From 2000 to 2001, he was a Visiting Professor with the Electrical and Computer Engineering Department, Henry Samueli School of Engineering, University of California, Irvine, CA, USA, working with reconfigurable antennas and microwave sensing of civil engineering structures. Director of the Catalan Research Foundation (2002-04), Director of the UPC-Telefonica Chair on Information Society Future Trends (2003-), Principal Investigator of the 2008-13 Spanish Sensing Lab Consolider Projet, General Director and then Secretary for Catalan Universities and Research (2011-2016), and research leader of the 2017-20 ComSense Lab Maria de Maeztu project. He has authored more than 200 scientific and technical papers, reports, and chapters in specialized volumes. His current research interests include antennas, electromagnetic scattering and imaging, system miniaturization for wireless, and sensing industrial and bio-applications from microwaves to $\mathrm{THz}$ frequencies.

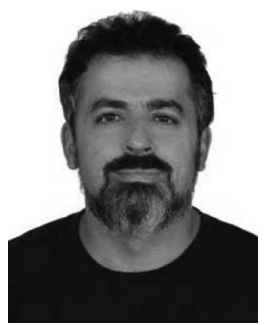

Bedri A. Cetiner is a Professor in the department of electrical engineering at Utah State University. From November 1999 to June 2000, he was with the University of California, Los Angeles, as a NATO Science Fellow. From June 2000 to June 2004, he worked as a research scientist at the ECE department of University of California, Irvine. From July 2004 until July of 2007, he worked as an Assistant Professor in the Department of Space Science and Engineering, Morehead State University, Kentucky. In August 2007, he joined Utah State University. He is also Founder, President and CEO of i5 Technologies Inc., Logan, UT. His research focuses on the applications of micro-nano technologies to a new class of micro-/millimeter-wave circuits and systems, and intelligent wireless communications systems with an emphasis on multifunctional reconfigurable antenna equipped MIMO systems. He is the Principal Inventor of nine patented technologies in the area of wireless communications. 